\title{
Exosomal miRNA signatures of pancreatic lesions
}

\author{
Caterina Vicentini ${ }^{1 \dagger}$, Federica Calore ${ }^{2 \dagger}$, Giovanni Nigita ${ }^{2}$, Paolo Fadda ${ }^{2}$, Michele Simbolo ${ }^{3}$, Nicola Sperandio ${ }^{1}$, \\ Claudio Luchini ${ }^{3}$, Rita T. Lawlor ${ }^{1}$, Carlo Maria Croce ${ }^{2}$, Vincenzo Corbo ${ }^{1,3}$, Matteo Fassan ${ }^{1,4^{*}}$ (i) and Aldo Scarpa ${ }^{1,3}$
}

\begin{abstract}
Background: Pancreatic and peri-pancreatic neoplasms encompass a variety of histotypes characterized by a heterogeneous prognostic impact. miRNAs are considered efficient candidate biomarkers due to their high stability in tissues and body fluids. We applied Nanostring profiling of circulating exosomal miRNAs to distinct pancreatic lesions in order to establish a source for biomarker development.

Methods: A series of 140 plasma samples obtained from patients affected by pancreatic ductal adenocarcinoma (PDAC, $n=58$ ), pancreatic neuroendocrine tumors (PanNET, $n=42$ ), intraductal papillary mucinous neoplasms (IPMN, $n=20$ ), and ampulla of Vater carcinomas (AVC, $n=20$ ) were analyzed. Comprehensive miRNA profiling was performed on plasma-derived exosomes. Relevant miRNAs were validated by qRT-PCR and in situ hybridization (ISH).

Results: Lesion specific miRNAs were identified through multiple disease comparisons. Selected miRNAs were validated in the plasma by qRT-PCR and at tissue level by ISH. We leveraged the presence of clinical subtypes with each disease cohort to identify miRNAs that are differentially enriched in aggressive phenotypes.

Conclusions: This study shows that pancreatic lesions are characterized by specific exosomal-miRNA signatures. We also provide the basis for further explorations in order to better understand the relevance of these signatures in pancreatic neoplasms.
\end{abstract}

Keywords: Pancreatic lesions, Circulating miRNAs, Early biomarkers, Exosomes, NanoString profiling

\section{Background}

Pancreatic ductal adenocarcinoma (PDAC) represents approximately the $90 \%$ of all pancreatic cancers and is currently the third leading cause of cancer deaths in the United States with a reported 5-year survival rate of $8 \%$ [1]. Early symptoms of PDAC are non-specific, therefore more than half patients are diagnosed at a late stage when the tumor is unresectable $[2,3]$.

The second most common neoplasm of the pancreas is pancreatic neuroendocrine tumor (PanNET), a rare epithelial

\footnotetext{
* Correspondence: matteo.fassan@unipd.it

${ }^{+}$Caterina Vicentini and Federica Calore shared first authorship

'ARC-NET Research Centre, University of Verona, Verona, Italy

${ }^{4}$ Department of Medicine (DIMED), Surgical Pathology and Cytopathology Unit, University of Padua, Via Aristide Gabelli 61, 35121 Padua, PD, Italy

Full list of author information is available at the end of the article
}

malignancy arising from pancreatic islet cells [4]. At present, surgical resection of PanNET with curative intent remains the most effective therapeutic option, with systemic therapies being largely ineffective for unresectable diseases [5]. Even if PDAC and PanNET represent the two most common pancreatic cancers, they show completely different genetic profiles $[6,7]$.

Several risk factors associated to PDAC development have been identified including genetic syndromes, smoking, alcohol and chronic pancreatitis (CP) [8, 9]. In particular, $\mathrm{CP}$ has been proposed as independent risk factor for pancreatic cancer development [10].

Three main PDAC precursor lesions have been identified: pancreatic intraepithelial neoplasia (PanIN), intraductal papillary mucinous neoplasm (IPMN) and mucinous

(c) The Author(s). 2020 Open Access This article is licensed under a Creative Commons Attribution 4.0 International License, which permits use, sharing, adaptation, distribution and reproduction in any medium or format, as long as you give appropriate credit to the original author(s) and the source, provide a link to the Creative Commons licence, and indicate if changes were made. The images or other third party material in this article are included in the article's Creative Commons licence, unless indicated otherwise in a credit line to the material. If material is not included in the article's Creative Commons licence and your intended use is not permitted by statutory regulation or exceeds the permitted use, you will need to obtain permission directly from the copyright holder. To view a copy of this licence, visit http://creativecommons.org/licenses/by/4.0/ The Creative Commons Public Domain Dedication waiver (http://creativecommons.org/publicdomain/zero/1.0/) applies to the data made available in this article, unless otherwise stated in a credit line to the data. 
cystic neoplasm (MCN), whose early detection and management could prevent progression to malignancy [11]. Several studies have focused their attention on the genetic heterogeneity of pancreatic precursor lesions in order to elucidate the timing of specific alterations in pancreatic tumorigenesis especially in the contest of IPMN and PDAC [12-14].

Ampulla of Vater carcinomas (AVC) comprise a percentage ranging from 15 to $30 \%$ of all pancreaticoduodenectomies and 10 to $20 \%$ of all tumor-related obstructions of the common bile duct $[15,16]$.

Although AVC show a better prognosis than PDAC, it remains a deadly disease with a mortality rate of $60 \%$ $[17,18]$. At present, diagnostic imaging is not able to distinguish between periampullary neoplasms such as periampullary PDAC or periampullary carcinoma of the duodenum and AVC [19].

The current therapeutic approaches for treating advanced pancreatic/peri-pancreatic cancers are poorly effective, and the known common biomarkers are inadequate for a reliable risk stratification as well as in the setting of early diagnosis. Therefore, new non-invasive strategies and more precise therapeutic targets are urgently needed to improve patients' management and outcome. MicroRNAs (miRNAs) are a class of endogenous, small (19 to 25 nucleotides), non-coding RNAs that modulate the expression of at least one third of protein-coding genes [20-24]. Moreover, miRNAs have been detected within body fluids, such as plasma, serum, urine and breast milk. The so-called "circulating microRNAs" can be found either encapsulated in cellsecreted vesicles or vesicle-free and instead be associated to AGO protein-positive ribonucleoprotein (RNP) particles [25]. It has been demonstrated that secreted miRNAs can be taken up by recipient cells and act as biologically active molecules [26, 27].

It has been widely reported in literature the aberrant miRNAs expression in human cancers including PDAC, its precursor lesions, PanNETs and AVCs both on tissue and biofluids [28-32]. Many genomic aberrations have been associated to the differential expression of miRNAs between normal and malignant tissues as chromosomal alterations, DNA point mutations, epigenetic mechanisms and miRNA processing machinery alterations [21].

On these grounds, the aim of this study was to identify circulating miRNAs able in discriminating the different histotypes of pancreatobiliary neoplasms. Furthermore, we also sought to identify miRNAs differentially expressed in the same histotype, based on biological and clinical differences. In particular, we leveraged the presence of metastatic and localized PDAC in our cohort to identify markers of dismal disease. To our knowledge, this is one of the most extensive study cohort of circulating miRNAs profiling among patients with different pancreatobiliary lesions using digital technology.

\section{Methods}

Pancreatic lesion patients and clinical samples

A series of 155 plasma samples obtained from 58 PDAC, 42 PanNETs, 20 IPMN, 20 AVC (Additional file 1), and $15 \mathrm{CP}$ patients were retrieved from the archives of the ARC-NET biobank of the Verona University Hospital. All experiments were performed in accordance with relevant guidelines and regulations. Clinicopathological features of PanNET, PDAC and AVC patients are reported in Table 1. Regarding IPMN samples, only 6 of 20 displayed associated invasive pancreatic ductal adenocarcinoma. All peripheral fasting blood samples were collected in 10-mL sodium heparin tubes (BD Vacutainer; Becton-Dickinson, Milan, Italy) and processed within $2 \mathrm{~h}$ by centrifugation at $2000 \mathrm{~g}$ at $4{ }^{\circ} \mathrm{C}$ for $10 \mathrm{~min}$. The plasma obtained was then transferred to new tubes and centrifuged at $3000 \mathrm{~g}$ at $4{ }^{\circ} \mathrm{C}$ for $10 \mathrm{~min}$ to remove platelet contamination. Final plasma preparations were carefully collected from the upper portion of the supernatant and stored in aliquots at $-80^{\circ} \mathrm{C}$.

\section{Preparation of exosomal RNA from pancreatic lesion plasma samples}

Plasma-derived exosomes were isolated as described in Casadei et al. [33] by using the ExoQuick system (System biosciences) according to the manufacturer's protocol. The quality and size of particles were determined by Nanosight, qRT-PCR and western blot analyses (Additional file 2). The exosomal pellet was resuspended in $1 \mathrm{ml}$ Trizol (Life Technologies), and total RNA was isolated with Norgen RNA clean-up and concentration kit (Norgen BioTek), following the provided instructions. Exosomal RNA was eluted in $20 \mu \mathrm{l}$ of $\mathrm{H}_{2} \mathrm{O}$; the average RNA yield per group per $1 \mathrm{ml}$ of plasma was: for the CP group $\sim 4 \mathrm{ng} / \mathrm{ml}$, for IPMN $\sim 6 \mathrm{~nm} /$ $\mathrm{ml}$, for PDAC $\sim 6.3 \mathrm{ng} / \mathrm{ml}$, for AVC $\sim 20 \mathrm{ng} / \mathrm{ml}$, for PanNET $\sim 4.7 \mathrm{ng} / \mathrm{ml}$. Nanodrop 1000 spectrophotometer (Thermo Scientific) was used to exclude within our samples the presence of $230 \mathrm{~nm}$ peaks, due to organics compounds or chaotropic salts, which could represent a potential issue for the ligase enzymatic step during NanoString profiling.

\section{Nanoparticle tracking analysis (NTA)}

Concentration and particle size distribution in plasmaderived exosomal samples were determined by NTA using NanoSight NS300 system (Malvern Technologies, Malvern, UK). Nanosight was configured with a $532 \mathrm{~nm}$ green laser, a high sensitivity scientific CMOS camera, and samples were loaded by using a syringe. Samples were diluted in particle-free PBS (Sigma) to acceptable concentrations based off of the manufacturer's recommendation. Sample data files were acquired under continuous flow (flow rate $=25-50$ ) and at room temperature. Samples were recorded for $3 \times 60$ s successive videos and were captured with a camera level of 12 . 
Table 1 Clinicopathological characteristics of considered PDAC, PanNET and AVC

\begin{tabular}{|c|c|c|c|}
\hline Variable & PDAC $(n=55)$ & PanNET $(n=42)$ & $\operatorname{AVC}(n=19)^{b}$ \\
\hline \multicolumn{4}{|l|}{ Gender } \\
\hline F & $22(40 \%)$ & 16 (38\%) & 10 (53\%) \\
\hline M & $33(60 \%)$ & $26(62 \%)$ & $9(47 \%)$ \\
\hline \multirow[t]{2}{*}{ Age (years) } & $64.5 \pm 10,5$ & $51.1 \pm 13.65$ & $63.4 \pm 11.18$ \\
\hline & (median 67.0) & (median 49.5) & (median 63) \\
\hline \multicolumn{4}{|l|}{ Primary tumor } \\
\hline $\mathrm{T} 1$ & $5(9 \%)$ & $20(47 \%)$ & $3(15 \%)$ \\
\hline $\mathrm{T} 2$ & $25(46 \%)$ & 15 (36\%) & $6(30 \%)$ \\
\hline T3 & $9(16 \%)$ & $7(17 \%)$ & $9(45 \%)$ \\
\hline T4 & $6(11 \%)$ & $0(0 \%)$ & $0(0 \%)$ \\
\hline an.a. & $10(18 \%)$ & $0(0 \%)$ & $1(5 \%)$ \\
\hline \multicolumn{4}{|c|}{ Regional lymph nodes } \\
\hline NO & $3(6 \%)$ & $22(52 \%)$ & $7(35 \%)$ \\
\hline $\mathrm{N} 1$ & 15 (27\%) & $12(29 \%)$ & $7(35 \%)$ \\
\hline N2 & $21(38 \%)$ & $0(0 \%)$ & $4(20 \%)$ \\
\hline n.a. & $16(29 \%)$ & $8(19 \%)$ & $1(5 \%)$ \\
\hline \multicolumn{4}{|c|}{ Distant metastasis } \\
\hline MO & $38(69 \%)$ & 40 (95\%) & 18 (90\%) \\
\hline M1 & $11(20 \%)$ & $2(5 \%)$ & $1(10 \%)$ \\
\hline n.a. & $6(11 \%)$ & $0(0 \%)$ & $0(0 \%)$ \\
\hline \multicolumn{4}{|l|}{ Clinical stage } \\
\hline । & $3(6 \%)$ & $11(26 \%)$ & $6(30 \%)$ \\
\hline$\|$ & 15 (27\%) & $11(26 \%)$ & $0(0 \%)$ \\
\hline III & $20(36 \%)$ & $10(24 \%)$ & $12(60 \%)$ \\
\hline IV & $11(20 \%)$ & $2(5 \%)$ & $1(5 \%)$ \\
\hline n.a. & $6(11 \%)$ & $8(19 \%)$ & $0(0 \%)$ \\
\hline \multicolumn{4}{|l|}{ Grade } \\
\hline G1 & $0(0 \%)$ & $29(69 \%)$ & $2(10 \%)$ \\
\hline G2 & $25(45.5 \%)$ & $13(31 \%)$ & $12(60 \%)$ \\
\hline G3 & $14(25.5 \%)$ & $0(0 \%)$ & $4(20 \%)$ \\
\hline n.a. & $16(29 \%)$ & $0(0 \%)$ & $1(5 \%)$ \\
\hline \multicolumn{4}{|c|}{ Lymphovascular invasion } \\
\hline absent & $1(2 \%)$ & $17(40.5 \%)$ & $3(15 \%)$ \\
\hline present & $38(69 \%)$ & $17(40.5 \%)$ & $15(75 \%)$ \\
\hline n.a. & $16(29 \%)$ & $8(19 \%)$ & $1(5 \%)$ \\
\hline \multicolumn{4}{|c|}{ Perineural invasion } \\
\hline absent & $0(0 \%)$ & $22(52 \%)$ & $5(25 \%)$ \\
\hline present & $39(71 \%)$ & $12(29 \%)$ & $13(65 \%)$ \\
\hline n.a. & $16(29 \%)$ & $8(19 \%)$ & $1(5 \%)$ \\
\hline
\end{tabular}

${ }^{a}$ n.a (not available data): patients who did not undergo radical surgery; ${ }^{b}$ One out of 20 AVCs is represented by an adenomatous lesion with high-grade dysplasia (this case has not been reported in the table)
Data were analyzed using NTA 3.1.5 software with a detection threshold of 3-4.

\section{NanoString nCounter assay and data analysis}

A total of 155 samples were processed with Nanostring nCounter ${ }^{\oplus}$ Human v3 miRNA Expression Assay. NanoString analysis was performed as described in Drusco et al. [34]. Briefly, $3.5 \mu \mathrm{L}$ of exosomal RNA were annealed with multiplexed DNA tags (miR-tag) and bridges target specifics. Mature miRNAs were then bond to specific miR-tags using a Ligase enzyme and all the tags in excess were removed by enzyme clean-up step. The tagged microRNAs product was diluted 1 to 5 and $5 \mu \mathrm{L}$ were combined with $20 \mu \mathrm{L}$ of Reported Probes in hybridization buffer and $5 \mu \mathrm{L}$ of Capture probes. The overnight hybridization (16 to $20 \mathrm{~h}$ ) at $65^{\circ} \mathrm{C}$ allowed to complex probes sequence specific with targets. Probe excess was removed using two-step magnetic beads-based purification on an automated fluidic handling system (nCounter Prep Station) and target/probe complexes were immobilized on the cartridge for data collection. The nCounter Digital Analyzer collected the data by taking images of immobilized fluorescent reporters in the sample cartridge with a CCD camera through a microscope objective lens. For each cartridge, a high-density scan encompassing 325 fields of view was performed. Images were processed internally into a digital format (RCC files). NanoString raw data were analyzed with nSolver ${ }^{\mathrm{TM}}$, a software provided by NanoString Technologies. Negative controls were used to perform background subtraction. Positive controls were used to perform technical normalization to adjust any lane-by-lane variability due to differences in hybridization, purification or binding. After technical normalization, the data were biologically normalized by calculating the geometric mean of the top 100 miRNAs in all samples, as recommended by NanoString. $p$-values were calculated using the LIMMA package (Linear Models for Microarray Data) from the Bioconductor $\mathrm{R}$ project. The $p$-values were adjusted for multiple testing using the Benjamini and Hochberg method [35] to control the False Discovery Rate (FDR). For each pairwise analysis, we considered all those significant $(p$-value $<0.05)$ and expressed $(\geq 20$ counts in at least one condition, as 20 counts is approximately the average of the expression of the negative controls in the NanoString panel) deregulated miRNAs for the downstream analysis.

\section{Quantitative real-time PCR}

The expression of an exosomal individual mature miRNA was assessed in triplicate by using the TaqMan Stem-loop miRNA assay, according to the manufacturer's protocol (ThermoFisher). For retro-trancription, $0.5 \mathrm{ng}$ of RNA were used per reaction in a final volume of $7.5 \mu \mathrm{l}$. For qRT-PCR, $1 \mu \mathrm{l}$ of $\mathrm{cDNA}$ was used per 
reaction. For RNA normalization, ath-miR-159a, osamiR-414, and cel-miR-248 synthetic oligos (Integrated DNA Technologies) were added to each sample right after the exosomal pellet was resuspended in Trizol. Normalization was performed with the $2^{-\Delta c t}$ method. Results were analyzed by using a two-tailed Student $t$ test.

\section{Western blot}

For the western blot analysis, exosomal proteins were isolated from $500 \mu \mathrm{l}$ of plasma (the yield of exosomal protein was $9 \mathrm{mg} / \mathrm{sample}$ ). The exosomal pellet was resuspended in RIPA buffer (Cell Signaling technology), supplemented with phosphatase and protease inhibitors (Roche), and incubated in ice for $20^{\prime}$. Samples were then harvested at 14,000 x g for $10^{\prime}$, and the supernatant collected in a new eppendorf. Protein concentration was determined by using Bradford Assay (Bio-Rad), following the manufacturer's instructions. $80 \mu \mathrm{g}$ of exosomal lysate were then loaded on a Criterion Tris- $\mathrm{HCl} 4-20 \%$ pre-cast gel (Bio-Rad), transferred onto a nitrocellulose membrane (Bio-Rad) and probed with antiAlix (1:1000), anti-TSG101 (1:1000), anti-Calnexin (Sigma) (1:2000), and anti-CD9 (Cell Signaling Technology) (1: 1000) primary antibodies, followed by isotype matched, horseradish-peroxidase-conjugated secondary antibodies. Finally, the proteins of interest were detected through chemi-luminescence reaction.

\section{miRNA in situ hybridization analysis (ISH)}

Locked nucleic acid (LNA) probes with complementarity to miR-4454, miR-106a-5p, and miR-17-5p were labelled with $5^{\prime}$-biotin and synthesized using Exiqon (Vedbaek, Denmark). Tissue sections were digested with ISH protease 1 (Ventana Medical Systems, Milan, Italy) and ISH was performed as we previously described [36]. Positive (U6; Exiqon) and negative scrambled LNA probes (Exiqon) were used as controls. Only cytoplasmic miRNA staining was retained for scoring purposes.

\section{Results}

Digital profiling identifies circulating miRNAs specific to the neoplastic state

Comprehensive miRNA profiling was performed in order to identify exosomal miRNAs differently expressed between pancreatic lesions (AVC, IPMN, PDAC and PanNET) and chronic pancreatitis (CP). Overall, we found 26, 23, 40 and 45 deregulated miRNAs between AVC vs CP, IPMN vs CP, PDAC vs CP and PanNET vs CP, respectively (Fig. 1 a-b-c-d). For each comparison, a linear fold change > 1.5 was used as threshold. Next, relevant miRNAs were filtered again considering only those with a number of counts greater than 20 (See Methods section, Table 2 and Additional file 3). In details, we found 5 deregulated miRNAs (3 upregulated and 2 downregulated miRNAs) between AVC and CP; 4 miRNAs between
IPMN and CP (3 upregulated and 1 downregulated miRNAs); 9 miRNAs between PDAC and CP (3 upregulated and 6 downregulated miRNAs) and 11 miRNAs between PanNET and CP (6 upregulated and 5 downregulated miRNAs) (Table 2 and Additional file 3).

Seven miRNAs were therefore selected for orthogonal validation by qRT-PCR on the same cohort of patients: miR-106-5p, miR-7975, miR-4454, miR-16-5p, miR-25-3p, miR-320e and miR-451a (Fig. 1 e-k and Table 2). In particular, since the NanoString assay is not able to discriminate between miR-106a-5p/miR-17-5p and miR-4454/ miR-7975, we decided at first to perform a qRT-PCR analysis for these four miRNAs in order to understand their specific expression contribution within selected sample groups. At first, we found a significant upregulation of miR-106-5p in AVC and IPMN compared to CP (Fig. 1e) while miR-17-5p was not differentially deregulated between the same lesions (data not shown). The expression of both miRNAs was also investigated between PanNET and CP showing no different between groups (data not shown). Than we validated the downregulation of miR-7975 and miR-4454 in PDAC compared to CP while only miR-4454 was found downregulated in the comparison between PanNET and CP (Fig. $1 \mathrm{f}-\mathrm{g}$ ). The upregulation of miR-16-5p was also validated by qRT-PCR in IPMN groups, while the upregulation of miR-25-3p and miR-451a and the downregulation of miR-320e were validated as deregulated in PanNET as compared to CP (Fig. $1 \mathrm{i}-\mathrm{j}-\mathrm{k}$ ).

\section{Lesion specific circulating miRNAs}

Differential miRNA profiling was also performed in order to identify relevant deregulated miRNAs between different pancreatic lesions (AVC vs PDAC, IPMN vs PDAC and PanNET vs PDAC). As reported in Fig. 2, we were able to identify 63 deregulated miRNAs between AVC and PDAC, 54 miRNAs between IPMN and PDAC and 16 miRNAs between PanNET and PDAC (Fig. 2 a-b-c). A linear fold change> 1.5 was applied as threshold for each comparison. After data filtering, we found 22 upregulated miRNAs in AVC compared to PDAC, 21 upregulated miRNAs in IPMN compared to PDAC and 7 deregulated miRNAs between PanNET and PDAC (4 upregulated and 3 downregulated miRNAs in PanNETs compared to PDAC) (Table 2 and Additional file 4). Selected miRNAs were than validated by qRT-PCR between different pancreatic lesions as reported in Additional file 3. Consistent with NanoString data, we found miR-17-5p and miR-106-5p significantly upregulated in AVC and IPMN as compared to PDAC (Fig. 2 d-e and Table 2). The upregulation of miR16-5p and miR-19-3p in IPMN compared to PDAC was also validated as reported in Fig. 2 (f-g) and Table 2.

We also focused on the most deregulated circulating miRNAs associated with different biological and clinical subtypes of the same histotype: metastatic PDAC Vs 

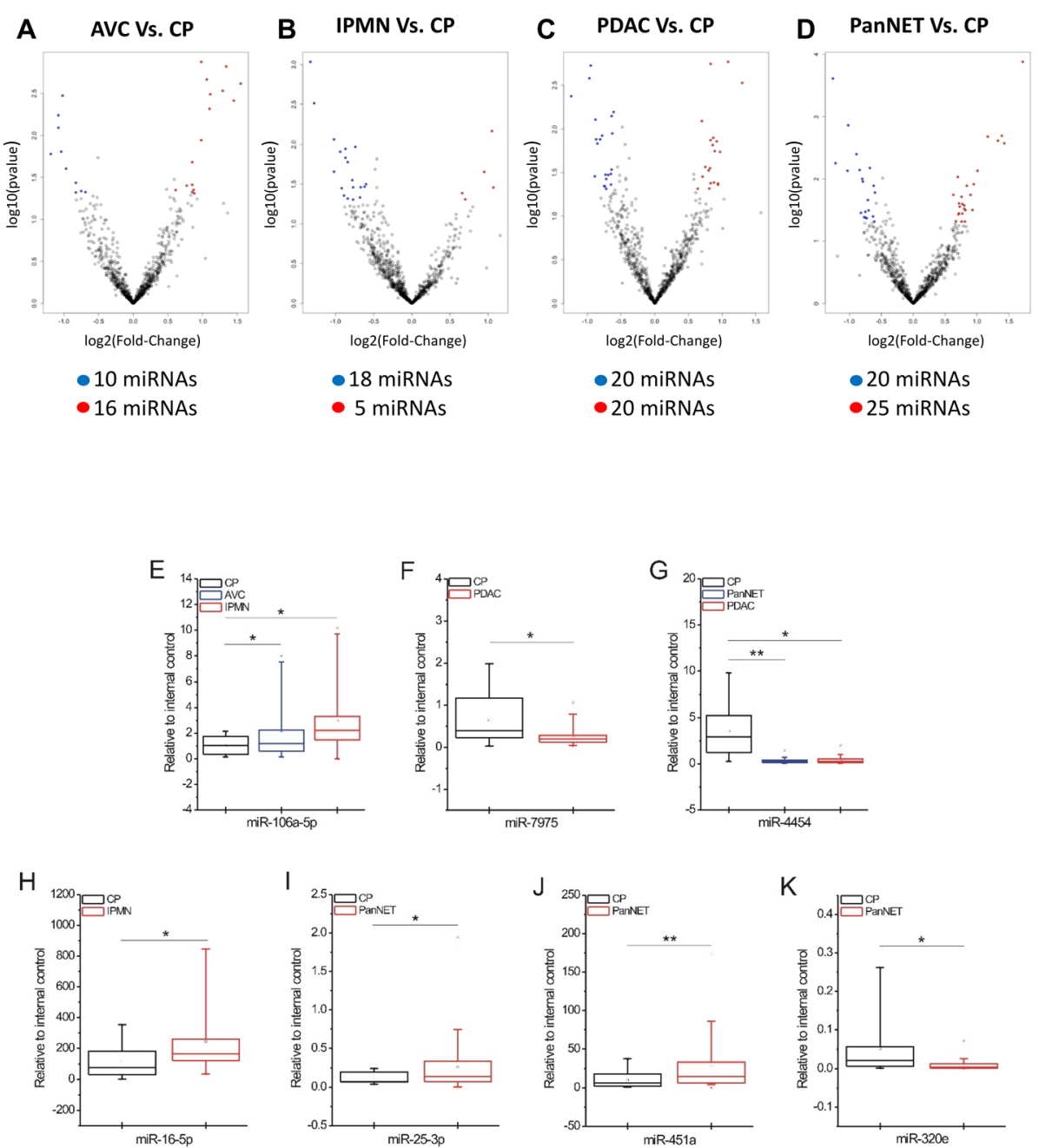

Fig. 1 Differential expression of circulating miRNAs in pancreatic lesions compared to chronic pancreatitis. a-b-c-d Volcano plots of miRNAs expression showing significant $(P<0.05)$ and deregulated (with a $\mid$ LinearFC $>1.5)$ miRNAs in each comparison. e-f-g-h-i-j-k NanoString results were validated by qRT-PCR analysis. The expression levels of the indicated miRNAs in the CP group (here considered as a control group) were compared with the expression of the same miRNAs within the indicated pancreatic cancer groups. The square inside the box plot indicates the mean value, whereas the " $x$ " outside the box indicates the 99 -percentile. Student's $t$-test was performed for statistical analysis. ${ }^{*}, 0.01<P \leq 0.05$; **, $0.001<P \leq 0.01$

localized PDAC (Met. PDAC vs Loc. PDAC), pancreatobiliary AVC vs intestinal AVC (PB AVC vs INT AVC) and IPMN with associated invasive adenocarcinoma and IPMN without associated invasive adenocarcinoma (IPMN-C VS IPMN) (Fig. 3). Using a linear fold change $>1.5$ as threshold, we identified overall 9 deregulated miRNAs between metastatic and localized PDAC, 11 miRNAs between PB AVC and INT AVC and 12 miRNAs between malignant IPMN and benign IPMN (Fig. 3 a-b-c). As for previous analysis, data were filtered again leading to the identification of 9 downregulated miRNAs in metastatic PDAC compared to the localized one, 11 deregulated miRNAs between pancreatobiliary and intestinal IPMN (6 upregulated and 5 downregulated miRNAs) and 12 miRNAs between malignant and benign IPMN (10 upregulated and 2 downregulated miRNAs) (Table 2 and Additional file 4).

\section{In situ analysis confirmed exosomal miRNA profiling}

To further support our findings, we performed in situ hybridization (ISH) assay on matched formalin-fixed paraffin-embedded (FFPE) tissue sections of CP, IPMN, AVC, PDAC patients and normal pancreas for miR4454, miR-106a-5p and miR-17-5p (Fig. 4). Despite ISH analyses lack the required sensitivity to identify subtle changes in expression levels of miRNA, they largely reflected qRT-PCR results. MiR-4454, which was significantly upregulated in CP compared to PDAC through qRT-PCR, showed the same expression trend by ISH 
Table 2 Summary of deregulated miRNAs from Nanostring profiling between pancreatic lesions

\begin{tabular}{|c|c|c|}
\hline Type of comparison & Upregulated miRNAs & Downregulated mRNAs \\
\hline AVC Vs. CP & $\begin{array}{l}\text { miR-106a-5p; miR-17-5p; } \\
\text { miR-520f-3p }\end{array}$ & miR-4454; miR-7975 \\
\hline IPMN Vs. CP & $\begin{array}{l}\text { miR-106a-5p; miR-17-5p; } \\
\text { miR-16-5p }\end{array}$ & miR-122-5p \\
\hline PDAC Vs. CP & $\begin{array}{l}\text { miR-372-3p; miR-140-3p; } \\
\text { miR-644a }\end{array}$ & $\begin{array}{l}\text { miR-1299; miR-146a-5p; } \\
\text { miR-148b-3p; miR-130a-3p; } \\
\text { miR-4454; miR-7975 }\end{array}$ \\
\hline PanNET Vs. CP & $\begin{array}{l}\text { miR-451a; miR-372-3p; } \\
\text { miR-106a-5p; miR-17-5p; } \\
\text { miR-25-3p; miR-644a }\end{array}$ & $\begin{array}{l}\text { miR-22-3p; miR-1246; } \\
\text { miR-4454; miR-7975; miR-320e }\end{array}$ \\
\hline ACV Vs. PDAC & $\begin{array}{l}\text { miR-106a-5p; miR-17-5p; } \\
\text { miR-2116-5p; miR-199a-3p; } \\
\text { miR-199b-3p; miR-342-3p; } \\
\text { miR-520d-5p; miR-527; } \\
\text { miR-518a-5p; miR-93-5p; } \\
\text { miR-30a-5p; miR-27b-3p; } \\
\text { miR-146a-5p; miR-451a; } \\
\text { miR-20a-5p; miR-20b-5p; } \\
\text { miR-185-5p; miR-520 h; } \\
\text { miR-25-3p; miR-1322; } \\
\text { miR-19a-3p; miR-302e }\end{array}$ & - \\
\hline IPMN Vs. PDAC & $\begin{array}{l}\text { miR-4454; miR-7975; } \\
\text { miR-2116-5p; miR-1910-5p; } \\
\text { miR-16-5p; miR-451a; } \\
\text { miR-19b-3p; miR-106a-5p; } \\
\text { miR-17-5p; miR-629-5p; } \\
\text { miR-376a-3p; miR-20a-5p; } \\
\text { miR-20b-5p; miR-26b-5p; } \\
\text { miR-93-5p; miR-25-3p; } \\
\text { miR-590-5p; miR-30e-5p; } \\
\text { miR-19a-3p; miR-608; } \\
\text { let-7b-5p }\end{array}$ & - \\
\hline PanNET Vs. PDAC & $\begin{array}{l}\text { miR-451a; miR-26b-5p; } \\
\text { miR-25-3p; miR-16-5p }\end{array}$ & $\begin{array}{l}\text { miR-1322; miR-1285-5p; } \\
\text { miR-320e }\end{array}$ \\
\hline Met. PDAC Vs. Loc. PDAC & $\begin{array}{l}\text { miR-106a-5p; miR-17-5p; } \\
\text { miR-342-3p; miR-20a-5p; } \\
\text { miR-20b-5p; miR-223-3p; } \\
\text { miR-16-5p; miR-19b-3p; } \\
\text { miR-130a-3p; miR-25-3p; } \\
\text { miR-451a }\end{array}$ & - \\
\hline PB AVC Vs. INT. AVC & $\begin{array}{l}\text { miR-579-3p; miR-422a; } \\
\text { miR-1253; miR-3144-3p; } \\
\text { miR-1268a; miR-190a-3p }\end{array}$ & $\begin{array}{l}\text { miR-3613-3p; miR-582-5p; } \\
\text { miR-1976; miR-885-5p; } \\
\text { miR-122-5p }\end{array}$ \\
\hline IPMN-C Vs. IPMN & $\begin{array}{l}\text { miR-1293; miR-450a-5p; } \\
\text { miR-433-5p; miR-324-5p; } \\
\text { miR-941; miR-499a-5p; } \\
\text { miR-4787-5p; miR-139-3p; } \\
\text { miR-516a-3p; miR-516b-3p; } \\
\text { miR-665 }\end{array}$ & miR-520f-3p; miR-126-3p \\
\hline
\end{tabular}

Significant (Pvalue $<0.05)$ and expressed $(\geq 20$ counts in at least one condition) deregulated (with a $\mid$ LinearFC $>1.5)$ miRNAs in each comparison. miRNAs validated by $\mathrm{QRT}$-PCR are indicated as bold text

(Fig. 4). MiR-106-5p and miR-17-5p qRT-PCR data also well matched with ISH experiments. As reported in Fig. 4, miR-106-5p was more expressed in IPMN and AVC tissue section as compared to PDAC and $\mathrm{CP}$, while the expression of miR-17-5p was more evident in AVC and IPMN compared to PDAC.

\section{Discussion}

In the last few years, many studies have highlighted the involvement of miRNAs in tumorigenesis and cancer progression supporting their possible role as new biomarkers for cancers [37]. Moreover, the identification of circulating miRNAs in the body fluids such as plasma/serum underline 


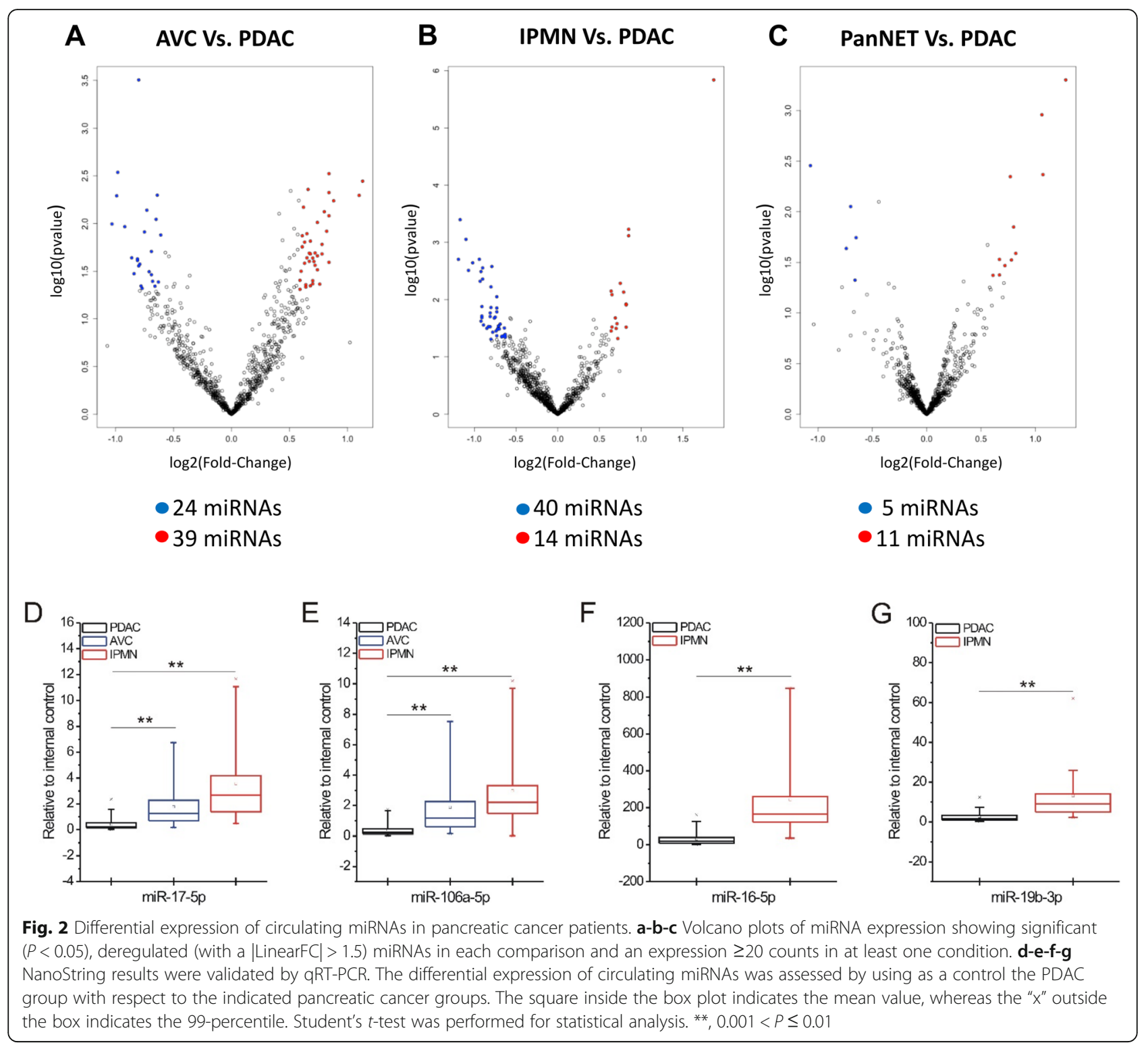

their potential application for detection, monitoring and predicting prognosis of cancer patients [24,38]. At present, several miRNA expression profiling studies identified miRNA signatures associated to clinico-pathological features in PDAC as staging, progression, prognosis and response to treatment both on tissues [39-43] and biofluids [44-49] while only few data have been published on miRNAs and gastroenteropancreatic neuroendocrine tumors [31, 50-52]. Genome-wide miRNA expression analyses were also performed on IPMN tissues [53-56] but only two studies evaluated blood-based miRNA expression in early stage PDAC patients versus controls $[45,55]$. As for IPMN, few information has been provided on circulating miRNAs and AVC, with plasma miR-192 up-regulation in periampullary carcinoma and its correlation with tumor stage and aggressiveness as the only identified biomarker [32, 57].
Despite this huge amount of data, miRNA expression profiling has not been introduced into the clinical practice, so far, maintaining radiological imaging and biopsy as the gold standards for tumor detection and diagnosis [58]. The causes of this failure may be found in the inconsistent results observed among studies or the unfeasibility to compare each other results due to different analytic methods, detection technologies or circulating components (e. g. plasma, serum or whole blood) analyzed [53]. On this ground, we applied digital profiling of circulating exosomal miRNAs to distinct pancreatic lesions in order to establish a bioresource for biomarker development. This is one of the most extensive exosomal miRNA study able to identify simultaneously peculiar miRNA signatures associate to different pancreatic lesions and within biological and clinical different subgroup of the same tumor histotype. 

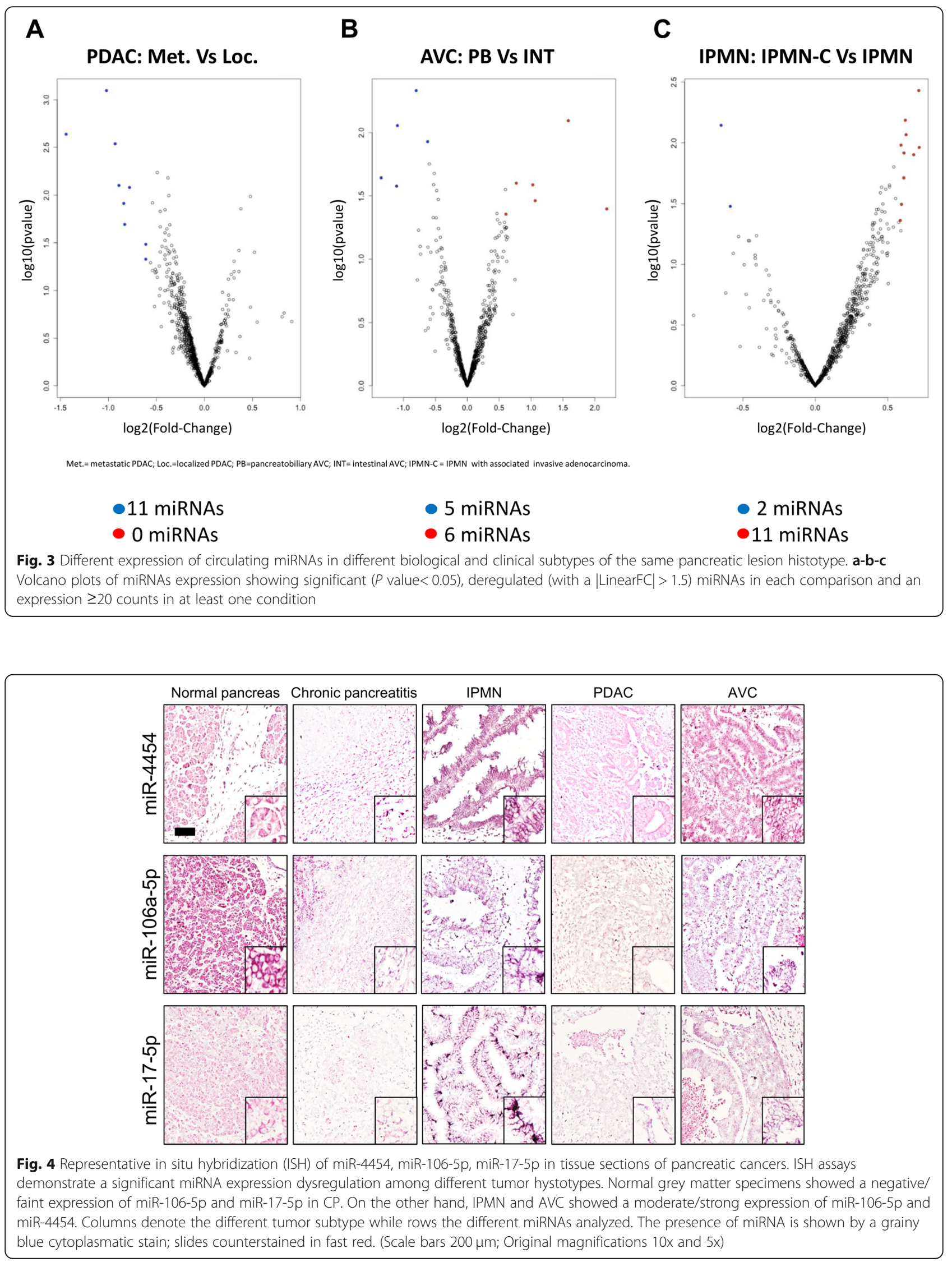
Recent evidences pointed out that exosomes represent a big bioresource of miRNAs, showing several advantages comparing to cell free miRNAs. First, exosomal miRNAs are protected from degradation even if in the presence of RNase and are stable under different storage conditions $[59,60]$. Secondly, it has been reported that they are representative of the matched parental tumor in terms of miRNA signatures $[61,62]$ and finally, it is known that malignant tissues secrete a higher amount of exosomes compared to the normal counterpart in biofluids such as plasma and urine [63, 64]. These peculiar features render exosomal miRNAs a promising candidate for miRNAs' profile studies.

In our study, plasma-derived exosomal RNA of 140 pancreatic lesions and 15 cases of chronic pancreatitis were processed by NanoString technology. Data analysis allowed us to select differentially expressed miRNAs in the majority of comparison between histotypes and $\mathrm{CP}$ patients. Selected miRNAs (miR-106-5p; miR-4454; miR-7975; miR-320e; miR-19b-3p; miR-16-5p and miR$17-5 p)$ were validated between groups by qRT-PCR underling the powerful of NanoString technique for miRNAs profiling, especially for small RNA quantities as in our case (Figs. 1 and 2). Three selected miRNAs (miR-4454; miR-106a-5p and miR-17-5p) were then validated also by in situ hybridization, which largely reflected the results of plasma profiling and showed that most of circulating miRNAs were derived from the epithelial components of the lesions.

For the first time miR-4454 was found significantly upregulated in the plasma of patients with chronic pancreatitis compared to PDAC and PanNET (Fig. 1g); notably, this result has been also validated by ISH on tissue samples, further corroborating its reliability (Fig. 4). In line with our findings, in the literature there is evidence supporting the role of miR-4454 in inflammation. For example, it has been reported in lung that miR-4454 targets include cytokines and matrix metalloproteinases that could have a relevant impact on pulmonary inflammation and fibrosis [65]. Moreover miR-4454 was also identified as mediators of facet cartilage degeneration promoting inflammatory, catabolic and cell death activity [66].

Next, we aimed to identify miRNAs associated to biological and clinical different subtypes within the same histotype. Since recent studies highlighted the role of exosomes in the formation of a premetastatic niche in the liver [67] and in tumor proliferation [68], we profiled, for the first time, exosomal miRNAs between localized and metastatic pancreatic ductal adenocarcinoma. Our analysis identified a signature of 11 miRNAs significantly downregulated in metastatic PDAC compared to localized disease (Table 2). Further functional studies should investigate the biological role of this signature as useful noninvasive tool able to monitor disease progression.
The current standard histopathologic evaluation is often not able to identify the precise origin of a periampullary lesions, including neoplasms from other subtypes as could appended for ampullary carcinomas [69]. Moreover, since AVC may be classified as pancreatobiliary or intestinal according to the histologic type of differentiation [70], we explored the possibility to identify peculiar exosomal miRNAs signatures able to discriminate between PDAC and AVC and within AVC subtypes. In particular, we found 22 exosomal miRNAs differently expressed between AVC and PDAC and 11 exosomal miRNAs between pancreatobiliary and intestinal AVC (Table 2). These signatures could represent an important bioresources for further validations aimed to discriminate between different lesions.

IPMN showed a wide histological spectrum ranging from benign adenomas to invasive carcinoma and represent an important precursor of aggressive pancreatic ductal adenocarcinoma. Since IPMN with an associated PDAC have a significantly worse prognosis compared to IPMN without PDAC and since the identification of this association is important especially prior the surgery [71], we investigated potential different exosomal miRNAs signature between these two entities identifying 13 differently expressed miRNAs between lesions (Table 2).

\section{Conclusion}

In this study we showed that pancreatic lesions are characterized by specific exosomal miRNAs signatures. We also provided a bioresource for future explorations aimed to understand the biological and clinical relevance of such signatures.

\section{Supplementary information}

Supplementary information accompanies this paper at https://doi.org/10. 1186/s12876-020-01287-y.

Additional file 1: Table S1. Summary of clinicopathological characteristics of pancreatic neoplasm patients.

Additional file 2: Figure S1. Quality and size analysis of pancreatic plasma-derived exosomes.

Additional file 3: Table S2. Deregulated miRNAs from Nanostring profiling associated to different pancreatic lesions.

Additional file 4: Table S3. Deregulated miRNAs from Nanostring profiling associated to biological and clinical different subtypes within the same histotype.

\section{Abbreviations}

PDAC: pancreatic ductal adenocarcinoma; PanNET: pancreatic neuroendocrine tumors; IPMN: intraductal papillary mucinous neoplasms; AVC: ampulla of Vater carcinomas; PanIN: pancreatic intraepithelial neoplasia; MCN: mucinous cystic neoplasm; CP: chronic pancreatitis; Met. PDAC: metastatic PDAC; Loc. PDAC: Localized PDAC; PB AVC: pancreatobiliary AVC; INT AVC: intestinal AVC; IPMN-C: IPMN with associated invasive adenocarcinoma 


\section{Acknowledgments}

We thank Alexander Ridenour and Alex Cornwell from The Ohio State University Analytical Cytometry Shared Resources for the support with Nanosight analysis. We thank S. Grimaldi and G. Bonizzato from ARC-NET Research Centre for the support with the collection of materials.

\section{Authors' contributions}

C.V., M.F., V.C., A.S., C.M.C., conceived the study. F.C., P.F., M.S., V.C., M. F, C. V designed the validation experiment. N.S. collected materials and clinical data. R.T.L. provided facilities. A.S., M.F., C.L. analyzed histopathological data. F.C., G.N., P.F. carried out NanoString profiling and raw data analysis. G. N, P.F. performed bioinformatic analyses. C.V., M.F., V.C., A.S., F. C, C.M.C. drafted the manuscript. All authors approved the final version of the manuscript.

\section{Funding}

This research was supported by Associazione Italiana Ricerca sul Cancro (AIRC, \# 12182 to AS, and \# 18718 to VC) and by NIH R35CA197706 and R01CA190740 to CMC in study design, data analysis, validation experiments design. The funding agencies had no role in the collection, analysis and interpretation of data and in the writing of the manuscript. NanoString nCounter and qRT-PCR analyses were performed at The Ohio State Genomics Shared Resources (GSR). All the Shared Resources at The Ohio State University that contributed to this paper were supported by the Cancer Center Support Grant P30CA016058.

\section{Availability of data and materials}

Supporting data and protocols are made available without restrictions from Prof. Aldo Scarpa.

\section{Ethics approval and consent to participate}

Materials used in the experiments have been collected under the Program 1885 with protocol 52438 approved on November 2010. The protocol includes written informed consent of the patient and were approved by the local ethics committee of the Integrated University Hospital Trust of Verona.

\section{Consent for publication}

Not applicable.

\section{Competing interests}

The authors have no competing interests to declare. The corresponding author of the present manuscript and the co-author Dr. Claudio Luchini are Editorial Board Member of BMC Gastroenterology.

\section{Author details}

${ }^{1}$ ARC-NET Research Centre, University of Verona, Verona, Italy. ${ }^{2}$ Department of Cancer Biology and Genetics and Comprehensive Cancer Center, Ohio State University, Columbus, Ohio, USA. ${ }^{3}$ Department of Diagnostics and Public Health, Section of Anatomical Pathology, University and Hospital Trust of Verona, Verona, Italy. ${ }^{4}$ Department of Medicine (DIMED), Surgical Pathology and Cytopathology Unit, University of Padua, Via Aristide Gabelli 61, 35121 Padua, PD, Italy.

Received: 1 October 2019 Accepted: 29 April 2020

Published online: 06 May 2020

\section{References}

1. Siegel RL, Miller KD, Jemal A. Cancer statistics, 2018. CA Cancer J Clin. 2018; 68(1):7.

2. Zhou B, Xu JW, Cheng YG, Gao JY, Hu SY, Wang L, et al. Early detection of pancreatic cancer: where are we now and where are we going? Int J Cancer. 2017;141(2):231.

3. Kamisawa T, Wood LD, Itoi T, Takaori K. Pancreatic cancer. Lancet. 2016; 388(10039):73.

4. Wolfgang $C L$, Herman JM, Laheru DA, Klein AP, Erdek MA, Fishman EK, et al. Recent progress in pancreatic cancer. CA Cancer J Clin. 2013;63(5):318.

5. Capelli P, Fassan M, Scarpa A. Pathology - grading and staging of GEP-NETs. Best Pract Res Clin Gastroenterol. 2012;26(6):705.

6. Cancer Genome Atlas Research Network. Electronic address aadhe, Cancer Genome Atlas Research N. Integrated Genomic Characterization of Pancreatic Ductal Adenocarcinoma. Cancer Cell. 2017;32(2):185.
7. Scarpa A, Chang DK, Nones K, Corbo V, Patch AM, Bailey P, et al. Wholegenome landscape of pancreatic neuroendocrine tumours. Nature. 2017; 543(7643):65.

8. Becker AE, Hernandez YG, Frucht H, Lucas AL. Pancreatic ductal adenocarcinoma: risk factors, screening, and early detection. World J Gastroenterol. 2014;20(32):11182

9. Pham A, Forsmark C. Chronic pancreatitis: review and update of etiology, risk factors, and management. F1000Res. 2018;7.

10. Sadr-Azodi O, Oskarsson V, Discacciati A, Videhult P, Askling J, Ekbom A. Pancreatic Cancer following acute pancreatitis: a population-based matched cohort study. Am J Gastroenterol. 2018;113(11):1711.

11. Riva G, Pea A, Pilati C, Fiadone G, Lawlor RT, Scarpa A, et al. Histo-molecular oncogenesis of pancreatic cancer: from precancerous lesions to invasive ductal adenocarcinoma. World J Gastrointest Oncol. 2018;10(10):317.

12. Amato E, Molin MD, Mafficini A, Yu J, Malleo G, Rusev B, et al. Targeted next-generation sequencing of cancer genes dissects the molecular profiles of intraductal papillary neoplasms of the pancreas. J Pathol. 2014;233(3):217.

13. Hosoda W, Chianchiano P, Griffin JF, Pittman ME, Brosens LA, Noe M, et al. Genetic analyses of isolated high-grade pancreatic intraepithelial neoplasia (HG-PanIN) reveal paucity of alterations in TP53 and SMAD4. J Pathol. 2017; 242(1):16.

14. Felsenstein M, Noe M, Masica DL, Hosoda W, Chianchiano P, Fischer CG, et al. IPMNs with co-occurring invasive cancers: neighbours but not always relatives. Gut. 2018;67(9):1652.

15. Pea A, Riva G, Bernasconi R, Sereni E, Lawlor RT, Scarpa A, et al. Ampulla of Vater carcinoma: molecular landscape and clinical implications. World 」 Gastrointest Oncol. 2018;10(11):370.

16. He J, Ahuja N, Makary MA, Cameron JL, Eckhauser FE, Choti MA, et al. 2564 resected periampullary adenocarcinomas at a single institution: trends over three decades. HPB (Oxford). 2014;16(1):83.

17. Albores-Saavedra J, Schwartz AM, Batich K, Henson DE. Cancers of the ampulla of vater: demographics, morphology, and survival based on 5,625 cases from the SEER program. J Surg Oncol. 2009;100(7):598.

18. Mafficini A, Amato E, Cataldo I, Rusev BC, Bertoncello L, Corbo V et al. Ampulla of Vater carcinoma: sequencing analysis identifies TP53 status as a novel independent prognostic factor and potentially actionable ERBB, PI3K. and WNT pathways gene mutations. Ann Surg. 2018;267(1):149.

19. Nikolaidis P, Hammond NA, Day K, Yaghmai V, Wood CG 3rd, Mosbach DS, et al. Imaging features of benign and malignant ampullary and periampullary lesions. Radiographics. 2014;34(3):624.

20. Di Leva G, Garofalo M, Croce CM. MicroRNAs in cancer. Annu Rev Pathol. 2014;9:287.

21. Di Leva G, Croce CM. miRNA profiling of cancer. Curr Opin Genet Dev. 2013; 23(1):3.

22. Medina PP, Slack FJ. microRNAs and cancer: an overview. Cell Cycle. 2008; 7(16):2485.

23. Xi Y, Nakajima G, Gavin E, Morris CG, Kudo K, Hayashi K, et al. Systematic analysis of microRNA expression of RNA extracted from fresh frozen and formalin-fixed paraffin-embedded samples. RNA. 2007:13(10):1668.

24. Mitchell PS, Parkin RK, Kroh EM, Fritz BR, Wyman SK, Pogosova-Agadjanyan EL, et al. Circulating microRNAs as stable blood-based markers for cancer detection. Proc Natl Acad Sci U S A. 2008;105(30):10513.

25. Turchinovich A, Tonevitsky AG, Burwinkel B. Extracellular miRNA: a collision of two paradigms. Trends Biochem Sci. 2016;41(10):883.

26. Katakowski $\mathrm{M}$, Buller $\mathrm{B}$, Wang $\mathrm{X}$, Rogers $\mathrm{T}$, Chopp M. Functional microRNA is transferred between glioma cells. Cancer Res. 2010;70(21):8259.

27. Aucher A, Rudnicka D, Davis DM. MicroRNAs transfer from human macrophages to hepato-carcinoma cells and inhibit proliferation. J Immunol. 2013;191(12):6250

28. Zhang Y, Li M, Wang H, Fisher WE, Lin PH, Yao Q, et al. Profiling of 95 microRNAs in pancreatic cancer cell lines and surgical specimens by realtime PCR analysis. World J Surg. 2009:33(4):698.

29. Yu J, Li A, Hong SM, Hruban RH, Goggins M. MicroRNA alterations of pancreatic intraepithelial neoplasias. Clin Cancer Res. 2012;18(4):981.

30. Permuth-Wey J, Chen DT, Fulp WJ, Yoder SJ, Zhang Y, Georgeades C, et al. Plasma MicroRNAs as novel biomarkers for patients with Intraductal papillary mucinous neoplasms of the pancreas. Cancer Prev Res (Phila). 2015;8(9):826.

31. Thorns C, Schurmann C, Gebauer N, Wallaschofski H, Kumpers C, Bernard V, et al. Global microRNA profiling of pancreatic neuroendocrine neoplasias. Anticancer Res. 2014;34(5):2249. 
32. Sandhu V, Bowitz Lothe IM, Labori KJ, Lingjaerde OC, Buanes T, Dalsgaard AM, et al. Molecular signatures of mRNAs and miRNAs as prognostic biomarkers in pancreatobiliary and intestinal types of periampullary adenocarcinomas. Mol Oncol. 2015;9(4):758.

33. Casadei L, Calore F, Creighton CJ, Guescini M, Batte K, Iwenofu OH, et al Exosome-derived miR-25-3p and miR-92a-3p stimulate Liposarcoma progression. Cancer Res. 2017;77(14):3846.

34. Drusco A, Fadda P, Nigita G, Fassan M, Bottoni A, Gardiman MP, et al. Circulating Micrornas Predict Survival of Patients with Tumors of Glial Origin. EBioMedicine. 2018;30:105

35. Benjamini $Y$, Hochberg Y. Controlling the false discovery rate: a practical and powerful approach to multiple testing. J Royal Stat Soc Series B. 1995; 57:289-300.

36. Carotenuto P, Fassan M, Pandolfo R, Lampis A, Vicentini C, Cascione L, et al. Wnt signalling modulates transcribed-ultraconserved regions in hepatobiliary cancers. Gut. 2017;66(7):1268.

37. Berindan-Neagoe I, Monroig Pdel C, Pasculli B, Calin GA. MicroRNAome genome: a treasure for cancer diagnosis and therapy. CA Cancer J Clin. 2014;64(5):311

38. Hollis M, Nair K, Vyas A, Chaturvedi LS, Gambhir S, Vyas D. MicroRNAs potential utility in colon cancer: early detection, prognosis, and chemosensitivity. World J Gastroenterol. 2015;21(27):8284.

39. Jamieson NB, Morran DC, Morton JP, Ali A, Dickson EJ, Carter CR, et al. MicroRNA molecular profiles associated with diagnosis, clinicopathologic criteria, and overall survival in patients with resectable pancreatic ductal adenocarcinoma. Clin Cancer Res. 2012;18(2):534.

40. Giovannetti E, Funel N, Peters GJ, Del Chiaro M, Erozenci LA, Vasile E, et al. MicroRNA-21 in pancreatic cancer: correlation with clinical outcome and pharmacologic aspects underlying its role in the modulation of gemcitabine activity. Cancer Res. 2010;70(11):4528.

41. Papaconstantinou IG, Manta A, Gazouli M, Lyberopoulou A, Lykoudis PM, Polymeneas $\mathrm{G}$, et al. Expression of microRNAs in patients with pancreatic cancer and its prognostic significance. Pancreas. 2013;42(1):67.

42. Giovannetti $\mathrm{E}$, van der Velde A, Funel N, Vasile $\mathrm{E}$, Perrone $\mathrm{V}$, Leon LG, et al. High-throughput microRNA (miRNAs) arrays unravel the prognostic role of MiR-211 in pancreatic cancer. PLoS One. 2012;7(11):e49145.

43. Vila-Navarro E, Vila-Casadesus M, Moreira L, Duran-Sanchon S, Sinha R, Gines A, et al. MicroRNAs for detection of pancreatic Neoplasia: biomarker discovery by next-generation sequencing and validation in 2 independent cohorts. Ann Surg. 2017;265(6):1226.

44. Schultz NA, Dehlendorff C, Jensen BV, Bjerregaard JK, Nielsen KR, Bojesen SE, et al. MicroRNA biomarkers in whole blood for detection of pancreatic cancer. JAMA. 2014;311(4):392.

45. Ganepola GA, Rutledge JR, Suman P, Yiengpruksawan A, Chang DH. Novel blood-based microRNA biomarker panel for early diagnosis of pancreatic cancer. World J Gastrointest Oncol. 2014;6(1):22.

46. Wang J, Raimondo M, Guha S, Chen J, Diao L, Dong X, et al. Circulating microRNAs in pancreatic juice as candidate biomarkers of pancreatic Cancer. J Cancer. 2014:5(8):696.

47. Liu R, Chen X, Du Y, Yao W, Shen L, Wang C, et al. Serum microRNA expression profile as a biomarker in the diagnosis and prognosis of pancreatic cancer. Clin Chem. 2012;58(3):610.

48. Kong X, Du Y, Wang G, Gao J, Gong Y, Li L, et al. Detection of differentially expressed microRNAs in serum of pancreatic ductal adenocarcinoma patients: miR-196a could be a potential marker for poor prognosis. Dig Dis Sci. 2011;56(2):602

49. Kishikawa T, Otsuka M, Ohno M, Yoshikawa T, Takata A, Koike K. Circulating RNAs as new biomarkers for detecting pancreatic cancer. World J Gastroenterol. 2015:21(28):8527.

50. Ruebel K, Leontovich AA, Stilling GA, Zhang S, Righi A, Jin L, et al. MicroRNA expression in ileal carcinoid tumors: downregulation of microRNA-133a with tumor progression. Mod Pathol. 2010;23(3):367.

51. Roldo C, Missiaglia E, Hagan JP, Falconi M, Capelli P, Bersani S, et al. MicroRNA expression abnormalities in pancreatic endocrine and acinar tumors are associated with distinctive pathologic features and clinical behavior. J Clin Oncol. 2006;24(29):4677.

52. Li SC, Essaghir A, Martijn C, Lloyd RV, Demoulin JB, Oberg K, et al. Global microRNA profiling of well-differentiated small intestinal neuroendocrine tumors. Mod Pathol. 2013;26(5):685.

53. Permuth-Wey J, Chen YA, Fisher K, McCarthy S, Qu X, Lloyd MC, et al. A genome-wide investigation of microRNA expression identifies biologically- meaningful microRNAs that distinguish between high-risk and low-risk intraductal papillary mucinous neoplasms of the pancreas. PLoS One. 2015; 10(1):e0116869.

54. Lubezky N, Loewenstein S, Ben-Haim M, Brazowski E, Marmor S, PasmanikChor $\mathrm{M}$, et al. MicroRNA expression signatures in intraductal papillary mucinous neoplasm of the pancreas. Surgery. 2013;153(5):663.

55. Caponi S, Funel N, Frampton AE, Mosca F, Santarpia L, Van der Velde AG, et al. The good, the bad and the ugly: a tale of miR-101, miR-21 and miR155 in pancreatic intraductal papillary mucinous neoplasms. Ann Oncol. 2013;24(3):734.

56. Matthaei $\mathrm{H}$, Schulick RD, Hruban $\mathrm{RH}$, Maitra A. Cystic precursors to invasive pancreatic cancer. Nat Rev Gastroenterol Hepatol. 2011;8(3):141.

57. Murali Manohar K, Sasikala M, Kvsrr Y, Sunil V, Talukdar R, Murthy H, et al Plasma microRNA192 in combination with serum CA19-9 as non-invasive prognostic biomarker in periampullary carcinoma. Tumour Biol. 2017:39(3): 1010428317695018

58. Lee ES, Lee JM. Imaging diagnosis of pancreatic cancer: a state-of-the-art review. World J Gastroenterol. 2014;20(24):7864.

59. Cheng $L$, Sharples RA, Scicluna BJ, Hill AF. Exosomes provide a protective and enriched source of miRNA for biomarker profiling compared to intracellular and cell-free blood. J Extracell Vesicles. 2014;3.

60. Ge Q, Zhou Y, Lu J, Bai Y, Xie X, Lu Z. miRNA in plasma exosome is stable under different storage conditions. Molecules. 2014;19(2):1568.

61. Zheng J, Yu L, Chen W, Lu X, Fan X. Circulating exosomal microRNAs reveal the mechanism of Fructus Meliae Toosendan-induced liver injury in mice. Sci Rep. 2018;8(1):2832.

62. Ogata-Kawata H, Izumiya M, Kurioka D, Honma Y, Yamada Y, Furuta K, et al. Circulating exosomal microRNAs as biomarkers of colon cancer. PLoS One. 2014;9(4):e92921.

63. Taylor DD, Gercel-Taylor C. MicroRNA signatures of tumor-derived exosomes as diagnostic biomarkers of ovarian cancer. Gynecol Oncol. 2008;110(1):13.

64. Andre F, Schartz NE, Movassagh M, Flament C, Pautier P, Morice P, et al. Malignant effusions and immunogenic tumour-derived exosomes. Lancet. 2002;360(9329):295.

65. Armstrong DA, Nymon AB, Ringelberg CS, Lesseur C, Hazlett HF, Howard L, et al. Pulmonary microRNA profiling: implications in upper lobe predominant lung disease. Clin Epigenetics. 2017:9:56.

66. Nakamura A, Rampersaud YR, Sharma A, Lewis SJ, Wu B, Datta P, et al. Identification of microRNA-181a-5p and microRNA-4454 as mediators of facet cartilage degeneration. JCI Insight. 2016;1(12):e86820.

67. Costa-Silva B, Aiello NM, Ocean AJ, Singh S, Zhang H, Thakur BK, et al. Pancreatic cancer exosomes initiate pre-metastatic niche formation in the liver. Nat Cell Biol. 2015;17(6):816.

68. Charrier A, Chen R, Chen L, Kemper S, Hattori T, Takigawa M, et al. Connective tissue growth factor (CCN2) and microRNA-21 are components of a positive feedback loop in pancreatic stellate cells (PSC) during chronic pancreatitis and are exported in PSC-derived exosomes. J Cell Commun Signal. 2014;8(2):147.

69. Westgaard A, Tafjord S, Farstad IN, Cvancarova M, Eide TJ, Mathisen O, et al. Pancreatobiliary versus intestinal histologic type of differentiation is an independent prognostic factor in resected periampullary adenocarcinoma. BMC Cancer. 2008:8:170.

70. Zhou H, Schaefer N, Wolff M, Fischer HP. Carcinoma of the ampulla of Vater: comparative histologic/immunohistochemical classification and follow-up. Am J Surg Pathol. 2004;28(7):875.

71. Sohn TA, Yeo CJ, Cameron JL, Hruban RH, Fukushima N, Campbell KA, et al. Intraductal papillary mucinous neoplasms of the pancreas: an updated experience. Ann Surg. 2004;239(6):788.

\section{Publisher's Note}

Springer Nature remains neutral with regard to jurisdictional claims in published maps and institutional affiliations. 